\title{
Moduli space theory for constant mean curvature surfaces immersed in space-forms
}

\author{
Alexandre GonÇAlves* And Karen Uhlenbeck
}

In this short article we describe a local parametrization of the space of solutions of the Gauss-Coddazzi equations for constant mean curvature immersions of a Riemann surface into space-forms in codimension 1. The parameter space are cohomology classes of the holomorphic tangent bundle of the surface.

\section{Introduction}

The study of constant mean curvature surfaces in a space-form has been an active field since the work of Hopf [10] in the 1920s and Liebmann in the years around 1900. The questions which are generally of interest are global questions of existence and uniqueness in complete 3-manifolds. We deal in this short paper on a question of existence and uniqueness with respect to the complex structure and the quadratic Hopf differential of a compact surface in a constant curvature 3-manifold which is not necessarily complete. Our final result applies only to the case of surfaces embedded in a local 3-dimensional space of constant curvature -1 , where the mean curvature constant $c$ satisfies $|c|<1$. However, the technique suggests some approaches to the more interesting cases, for example the work of Bryant [1] and Kenmotsu [2].

The Gauss-Codazzi equations for constant mean curvature immersions of a surface into a 3 -dimensional space-form are a $3 \times 3$ system of partial differential equations of mixed order. Once a complex structure is chosen, the equations break down into two equations. The Codazzi equation on the second fundamental form yields the Cauchy-Riemann equation for a holomorphic quadratic differential first noticed and used by Hopf [3]. The second is a real non-linear single elliptic equation for the length function of the metric which comes from the Gauss curvature equation.

These equations can be approached via a number of techniques in partial differential equations. In this short note, we improve upon results obtained

\footnotetext{
*Partially supported by FAPESP, Brazil, under grant 2006/02023-4.
} 
by assuming the Riemann surface structure and postulating a fixed quadratic differential representing the $(2,0)$ part of a second fundamental form as solving the Codazzi equations. This leaves the problem of solving the elliptic Gauss equation for the length function of the metric. By analyzing the Gauss and Codazzi equations together, we are able to reformulate the equations in a form which completely identifies all local solutions in the case of negative curvature. We prove that the moduli space of solutions to the Gauss-Codazzi equations for a Riemann surface of genus greater than one immersed with mean curvature constant and less than 1, in a not necessarily complete 3 -manifold of constant curvature -1 , is parameterized by cohomology classes of $(-1,1)$ differentials.

The result is similar and proved in the same fashion as the results in gauge theory in a paper of the first author [4]. In fact, the details of how the computations change with the change in basepoint $g$ is complicated, but it is familiar to geometers from the variational formulation of the Yamabe problem and will not be repeated. An abstract proof could be constructed along the lines of the convexity theory used in the gauge theory literature to describe bundle extensions by Bradlow and Garcia-Prada [5] and Daskalopolous et al. [6]. Also, as presented here, the construction is not as natural as it would be, if viewed from the point of view of these authors. However, the convexity theory fails in cases of positive curvature, whereas the reformulation of the Gauss-Codazzi equations as the Euler-Lagrange equations of a single variational problem has the potential to contribute to the more interesting cases of zero or even positive curvature.

It is entertaining to note that the second author came across these equations more than 20 years ago in looking at the possibility that minimal surfaces could be used to parameterize quasi-Fuchsian hyperbolic 3manifolds [7]. Although there is a fairly good existence theory, it seems as if uniqueness is unlikely [8].

\section{The variational formulation}

We assume in this note that $X$ is a surface immersed with mean curvature $c$ in a 3-dimensional manifold $N$ of constant sectional curvature $k$. We do not assume that this 3 -manifold is complete. The induced metric and second fundamental form of $X \subset N$ satisfy a system of Gauss-Codazzi equations which can be analyzed in a series of steps as follows.

1. The induced metric on $X$ is of the form $h=h_{z \bar{z}} d z d \bar{z}$, where $(z, \bar{z})$ are local complex coordinates in a complex structure $X_{\sigma}$ on $X$. 
2. The second fundamental form $\gamma$ has the structure

$$
\gamma=\alpha_{z z}(d z)^{2}+c h_{z \bar{z}} d z d \bar{z}+\alpha_{\bar{z} \bar{z}}(d \bar{z})^{2}
$$

Here $c$ is the (constant) mean curvature. $\gamma$ is symmetric with respect to the metric $h$, which implies the relationship

$$
\alpha_{z z}=\bar{\alpha}_{\bar{z} \bar{z}}
$$

3. The $(2,0)$ part of $\gamma$ is a holomorphic quadratic differential $\alpha$ on $X$,

$$
\bar{\partial} \alpha=0
$$

4. The induced (scalar) curvature $K$ of $X$ satisfies the Gauss equation.

$$
K=k+c^{2}-2|\alpha|^{2} .
$$

Here the norm of $\alpha$ is assumed to be taken with respect to the metric $h$. By rescaling, we may assume that $k=(-1,0,1)$. Our main results pertain to the case $\lambda=k+c^{2}<0$.

The solution to steps $1-3$ is completely understood, so an automatic procedure would be to fix the Riemann surface, the curvatures and the $(2$, 0 ) part $\alpha$ of the second fundamental form and attempt to solve the Gauss equation for the metric. There is a variational formulation of this problem. This last step results in an equation which can be solved for small $\alpha$ by the techniques of Kazden and Warner [9]. However, a slightly different variational problem arises when we solve for the second fundamental form and the metric in one step.

For convenience, we combine in a suggestive notation $\lambda=k+c^{2}$.

Fix the Riemann surface. We will choose as a base point the constant curvature metric on $X$ and a holomorphic quadratic differential. Let $\beta_{0}$ be the $(-1,1)$ form on $X$ which is dual to the chosen holomorphic differential in the constant curvature metric. Note that the identification between the $(2,0)$ and $(-1,1)$ forms depends on the metric. Now we compute with respect to an arbitrary element $\beta=\beta_{0}+\bar{\partial} f_{0}$ and fixed metric $g$ whose conformal class determines the Riemann surface. Let $K(g)$ denote the Gauss curvature of $g$. Calculations are always easier at the base metric, so we will want to have the freedom of changing it.

We call our functional $\mathcal{D}$ for Donaldson, as it is in reality a form of the Donaldson functional which appears in the construction of HermitianEinstein metrics in holomorphic vector bundles. Usually, there is no explicit 
formula for this functional. Due to the fact that we are in line bundles and are looking at an abelian gauge theory, the functional is for us explicit. Note that it is well defined up to an overall constant, which we have fixed by assuming the functional $\mathcal{D}$ is 0 on the constant curvature metric and the dual to the chosen holomorphic quadratic differential. Note that the holomorphic quadratic differential constructed from the variational principle is not the one that we started with.

Proposition 2.1. The metric and holomorphic quadratic differential pair

$$
(h, \alpha)=\left(e^{2 u} g, e^{2 u}\left(\beta^{*}+\partial f^{*}\right)\right)
$$

solve the Gauss-Codazzi system [(2.1) and (2.2)] if and only if $u: x \rightarrow c$, $f: x \rightarrow T^{1,0} x$ are critical maps for the functional:

$$
\mathcal{D}(u, f)=\iint\left[|\partial u|^{2}+K(g) u+e^{2 u}\left(-\lambda / 2+|\beta+\bar{\partial} f|^{2}\right)\right] d \mu+C(g) .
$$

Proof. This is a calculation. All the metrics, the covariant derivative $\partial=\bar{\partial}^{*}$ and the density are computed using the base metric $g$, although we suppress this in the statement of the theorem. We have included the constant $C(g, \beta)$, since we wish to make this computation $(g, \beta)$ independent. The value of $C(g)$ is determined by finding the value of the Donaldson functional at $g$ using our original choice of constant curvature metric and holomorphic quadratic differential.

Now the equation which arises from varying $f$ is a simple linear equation (in $f$, not $u$ )

$$
\bar{\partial}\left(e^{2 u}\left(\beta^{*}+\partial f^{*}\right)\right)=0
$$

This yields the holomorphic quadratic differential

$$
\alpha=e^{2 u}\left(\beta^{*}+\partial f^{*}\right)
$$

The equation obtained by varying $u$ is the equation

$$
0=-\partial^{*} \partial u+K(g)+e^{2 u}\left(-\lambda+2|\beta+\bar{\partial} f|^{2}\right) .
$$

Recall that in the new metric $h=e^{2 u} g$ the curvature $K(h)=e^{-2 u}(K(g)-$ $2 \partial \bar{\partial} u$ ), so that the equation which arises when varying $u$ is indeed the desired 
equation for mean curvature. It can be written as

$$
K(h)=\lambda-2 e^{-4 u}|\alpha|_{g}^{2}
$$

Note that the norm of $\alpha$ is correctly computed in the new metric $h$.

Corollary 2.2. Fix $\operatorname{vol}(X)=\iint e^{2 u} d \mu=T$. Then the critical points of

$$
\hat{\mathcal{D}}(u, f)=\iint|\partial u|^{2}+k(g) u+e^{2 u}|\beta+\bar{\partial} f|^{2} d \mu+C(g, \beta)
$$

with respect to the constraint provide solutions of the Gauss-Codazzi equations (3-4) with an unknown Lagrange multiplier $\lambda$.

The actual utilization of this minimization principle seems quite delicate. One would need to employ the Moser inequality carefully. Moreover, in many cases, one would be interested in saddle points rather than minima. However, this variational principle which fixes the cohomology class of $\beta$ has definite advantages over the one which fixes the holomorphic differential $\alpha$.

\section{Solutions for $\lambda<0$}

The main result of this short note is the following theorem.

Theorem 3.1. If $\lambda=k+c^{2}<0$, there exists a unique solution to the Gauss-Codazzi equations [(3) and (4)] for a fixed Riemann surface and a $(-1,1)$ cohomology class $[\beta]=\{\beta+\bar{\partial} f\}$.

Proof. We will show that for every solution to the Gauss-Codazzi equations with $\lambda=k+c^{2}<0$, the Hessian of $\mathcal{D}$ is positive definite. For small $[\beta]$, there will be a solution near a constant negative curvature metric of curvature $\lambda$ on $X$ which can be found using an implicit function theorem. Openness follows from the invertibility of the Hessian. Closedness is a rather easy calculation which we leave to the reader. It is similar to the proofs in the literature $[7,9]$.

Uniqueness follows since every solution can be connected to one with small cohomology class representative $[\beta]$. This leaves the important step of showing the positive definiteness of the Hessian to finish the proof.

Theorem 3.2. If $(u, f)$ is a critical point of $\mathcal{D}$, for a fixed Riemann surface and cohomology class $[\beta]$ with $\lambda<0$, then the Hessian is positive definite. 
Proof. We might as well make the calculation with the solution metric and holomorphic quadratic differential $\beta^{*}=\alpha$ as basepoint. (Here is where our comments about basepoint pay off. These calculations in gauge theory are where the idea for the proof comes from).

Since $(0,0)$ is a critical point, we have

$$
K(g)=\lambda-2|\beta|^{2}
$$

and

$$
\partial \beta=0
$$

The Hessian is easy to compute.

$$
H(v, f)=2 \iint\left[|\partial v|^{2}-v^{2} K(g)+4 v \operatorname{Re}\left\langle\beta, \partial f^{*}\right\rangle+\left\langle\bar{\partial} f, \partial f^{*}\right\rangle\right] d \mu .
$$

We note that $\partial \beta=0$, so we may replace

$$
2 \iint v \operatorname{Re}\left\langle\beta, \partial f^{*}\right\rangle d \mu=-2 \iint \operatorname{Re}\left\langle\partial v \otimes f^{*}, \beta\right\rangle d \mu .
$$

Also

$$
\frac{1}{2} \iint\left\langle\bar{\partial} f, \partial f^{*}\right\rangle d \mu=\frac{1}{2} \iint\left\langle\bar{\partial} f, \partial f^{*}\right\rangle-K(g)\left\langle f, f^{*}\right\rangle d \mu .
$$

We have $K(g)=\lambda-2|\beta|^{2}$, where $\lambda<0$. We rewrite the Hessian as

$$
\begin{aligned}
H(v, f)= & 2 \iint\left[|\partial v|^{2}+v^{2}\left(-\lambda+2|\beta|^{2}\right)+2 v \operatorname{Re}\left\langle\beta, \partial f^{*}\right\rangle\right. \\
& -2 \operatorname{Re}\left\langle\partial u \otimes f^{*}, \beta\right\rangle \\
& \left.+\frac{1}{2}\left(|\bar{\partial} f|^{2}+\frac{1}{2}|\partial f|^{2}+|f|^{2}\left(-\lambda+2|\beta|^{2}\right)\right)\right] d \mu \\
& >2 \iint\left[|\partial v|^{2}+2|v|^{2}|\beta|^{2}+\frac{1}{2}|\bar{\partial} f|^{2}+|f|^{2}|\beta|^{2}\right. \\
& -2|v||\beta||\bar{\partial} f|-2|\partial v||f||\beta|] d \mu \geq 0 .
\end{aligned}
$$

Note that we may allow $\lambda=0$ in all but extremely degenerate cases.

\section{References}

[1] R. Bryant, Surfaces of mean curvature one in hyperbolic space, Asterisque 154-155 (1987), 321-353.

[2] K. Kenmotsu, Surfaces of constant mean curvature, Translation of Math ematical Monographs, 221, Amer. Math Soc., 2004. 
[3] H. Hopf, 'Differential Geometry in the Large', Lecture Notes in Mathematis, 1000, SpringerVerlag, 1989.

[4] A. Gonçalves, An elliptic non-linear equation on a riemann surface, Diff. Geom. Appl. (2007), doi: 10.1016/j.difgeo.2007.02.006 at http://dx.doi.org

[5] S. Bradlow, and O. Garcia-Prado, Higher cohomology triples and holomorphic extensions, Comm. Anal. Geom. 3 (1995), 421-465.

[6] G. Daskalopolous, K. Uhlenbeck and R. Wentworth, Moduli of extensions of holomorphic bundles over Kaehler manifolds, Comm. Anal. Geom. 3 (1995), 479-522.

[7] K. Uhlenbeck, Closed minimal surfaces in hyperbolic 3 manifolds, in 'Seminar on Minimal Submanifolds', Ann. Math. Studies, 103, Princeton University Press, 1983, 147-168.

[8] J. Velling, Limits on prescribing the Hopf differential for minimal surfaces in $\boldsymbol{H}$, Commun Partial Diff. Equations 27 (2002), 2513-2525.

[9] J. Kazdan, and F. Warner, Curvature functions for compact 2-manifolds, Ann. Math. 99 (1964), 14-47.

[10] H. Hopf, Ueber Flachen mit einer Relation zwischen den Haupkreumungen, Math. Nachr. 4 (1951), 232-249.

\section{FFCLRP-DFM}

Av. Bandeirantes 3900, Monte Alegre

14040-901 RibeirÃo Preto, SP, Brazil

E-mail address: acasa@ffclrp.usp.br

Department of Mathematics

The University of Texas at Austin,

1 Univerisity Station, C1200

Austin, Texas 78712, USA

E-mail address: uhlen@math.utexas.edu

Received August 24, 2005 
